Mathematical Modelling and Analysis

Volume 22 Number 2, March 2017, 228-236

https://doi.org/10.3846/13926292.2017.1291455

(c) Vilnius Gediminas Technical University, 2017
Publisher: Taylor\&Francis and VGTU

http://www.tandfonline.com/TMMA

ISSN: 1392-6292

eISSN: $1648-3510$

\title{
Local Convergence of Jarratt-Type Methods with Less Computation of Inversion Under Weak Conditions
}

\author{
Ioannis K. Argyros ${ }^{a}$ and Santhosh George ${ }^{b}$ \\ ${ }^{a}$ Department of Mathematical Sciences, Cameron University \\ Lawton, 73505 OK, USA \\ ${ }^{b}$ Department of Mathematical and Computational Sciences, National Institute \\ of Technology Karnataka \\ 757025 India \\ E-mail(corresp.): sgeorge@nitk.ac.in
}

Received September 23, 2016; revised January 30, 2017; published online March 1, 2017

\begin{abstract}
We present a local convergence analysis for Jarratt-type methods in order to approximate a solution of a nonlinear equation in a Banach space setting. Earlier studies cannot be used to solve equations using such methods. The convergence ball and error estimates are given for these methods. Numerical examples are also provided in this study.

Keywords: multi-point methods, Banach space, convergence ball, local convergence, Jarratt-type methods.
\end{abstract}

AMS Subject Classification: 65D10; 65D99; 65G99; 47H17; 49M15.

\section{Introduction}

Let $X$ and $Y$ be Banach spaces and $F: \Omega \subset X \longrightarrow Y$ be a nonlinear continuously Fréchet-differentiable operator defined on a non-empty open convex subset of $X$. We are concerned with the problem of approximating a locally unique solution $x^{*}$ of the nonlinear equation

$$
F(x)=0 .
$$

Higher order methods like Jarratt method $[2,6,7]$ and fifth order method $[10,11]$ are considered for approximating the solution $x^{*}$ of $(1.1)$. But, for the convergence analysis of these methods, in addition to the assumptions on $F^{\prime}$ and $F^{\prime \prime}$ assumptions of the form (see $[2,6,7,10,11]$ ):

$$
\left\|F^{\prime \prime \prime}(x)-F^{\prime \prime \prime}(y)\right\| \leq L\|x-y\|, \quad x, y \in \Omega, L \geq 0
$$

or

$$
\left\|F^{\prime \prime \prime}(x)-F^{\prime \prime \prime}(y)\right\| \leq w(\|x-y\|), \quad x, y \in \Omega
$$


are required, where $w(z)$ is a nondecreasing continuous function for $z>0$ and $w(0)=0($ see $[10])$.

A typical example of (1.1) that does not satisfy (1.2) or (1.3) is the mixed Hammerstein type equation defined on $X=Y=C[0,1]$ by

$$
x(s)=\int_{0}^{1} Q(s, t)\left(x(t)^{\frac{3}{2}}+\frac{1}{2} x(t)^{2}\right) d t,
$$

where the kernel $Q$ is the Green's function defined on the interval $[0,1] \times[0,1]$ by

$$
Q(s, t)= \begin{cases}(1-s) t, & t \leq s \\ s(1-t), & s \leq t .\end{cases}
$$

Define $F: C[0,1] \longrightarrow C[0,1]$ by

$$
F(x)(s):=x(s)-\int_{0}^{1} G(s, t)\left(x(t)^{\frac{3}{2}}+\frac{1}{2} x(t)^{2}\right) d t
$$

and consider

$$
F(x)(s)=0 .
$$

Notice that $x^{*}(s)=0$ is one of the solutions of (1.1). Using (1.4), we obtain

$$
\left\|\int_{0}^{1} Q(s, t) d t\right\| \leq \frac{1}{8}
$$

Then, by (1.4)-(1.6), we have that

$$
\left\|F^{\prime}(x)-F^{\prime}(y)\right\| \leq \frac{1}{8}\left(\frac{3}{2}\|x-y\|^{\frac{1}{2}}+\|x-y\|\right) .
$$

Note that, $F^{\prime \prime \prime}$ is not Lipschitz. Hence the results in $[2,7,8,10,11]$ cannot be used to solve (1.5).

It is known ( [1]- [10]), that computational cost of inversion is very large, so many authors considered iterative methods with less computation of inversion $[1]-[10]$.

In this paper we study the local convergence of the three-step method defined for each $n=0,1,2 \ldots[10]$ by

$$
\begin{aligned}
& u_{n}=x_{n}-\frac{2}{3} F^{\prime}\left(x_{n}\right)^{-1} F\left(x_{n}\right), \\
& z_{n}=x_{n}-\left[I-\frac{3}{4}\left(I-\frac{3}{2} A\left(x_{n}\right)+\delta_{1} A\left(x_{n}\right)^{2}\right) A\left(x_{n}\right)\right] F^{\prime}\left(x_{n}\right)^{-1} F\left(x_{n}\right), \\
& x_{n+1}=z_{n}-\left[I-\frac{3}{2} A\left(x_{n}\right)+\delta_{2} A\left(x_{n}\right)^{2}\right] F^{\prime}\left(x_{n}\right)^{-1} F\left(z_{n}\right),
\end{aligned}
$$

where $x_{0}$ is an initial point, $\delta_{1}, \delta_{2} \in \mathbb{R}$ and $A\left(x_{n}\right)=F^{\prime}\left(x_{n}\right)^{-1}\left(F^{\prime}\left(u_{n}\right)-F^{\prime}\left(x_{n}\right)\right)$.

The almost sixth semilocal convergence order of method (1.7) was shown in [10] using the preceding Lipschitz-type conditions. However these results cannot apply to solve (1.5). The idea used in this paper can be used on other iterative methods $[1]-[9]$.

The rest of the paper is structured as follows: In Section 2 we present the local convergence analysis. We also provide a radius of convergence, computable error bounds and uniqueness result not given in the earlier studies [10,11]. Special cases and numerical examples are presented in the concluding Section 3. 


\section{Local Convergence}

The local convergence of method (1.7) that follows is based on some scalar functions. Let $w_{0}, w:[0,+\infty) \longrightarrow[0,+\infty), v:[0,+\infty) \longrightarrow(0,+\infty)$ be nondecreasing, continuous functions with $w_{0}(0)=w(0)=0$. Define parameter

$$
r_{0}=\sup \left\{t \geq 0: w_{0}(t)<1\right\}
$$

Moreover, define scalar functions on the interval $\left[0, r_{0}\right)$ by

$$
\begin{aligned}
g_{1}(t) & =\frac{1}{1-w_{0}(t)}\left(\int_{0}^{1} w((1-\theta) t) d \theta+\frac{1}{3} \int_{0}^{1} v(\theta t) d \theta\right) \\
g_{2}(t) & =\frac{\int_{0}^{1} w((1-\theta) t) d \theta}{1-w_{0}(t)}+\frac{3}{4}\left[\left(1+\frac{3}{2} \frac{w_{0}\left(g_{1}(t) t\right)+w_{0}(t)}{1-w_{0}(t)}\right.\right. \\
& \left.\left.+\left|\delta_{1}\right|\left(\frac{w_{0}\left(g_{1}(t) t\right)+w_{0}(t)}{1-w_{0}(t)}\right)^{2}\right) \frac{w_{0}\left(g_{1}(t) t\right)+w_{0}(t)}{1-w_{0}(t)}\right] \frac{\int_{0}^{1} v(\theta t) d \theta}{1-w_{0}(t)} \\
g_{3}(t) & =g_{2}(t)+\left(1+\frac{3}{2} \frac{w_{0}\left(g_{1}(t) t\right)+w_{0}(t)}{1-w_{0}(t)}\right. \\
& \left.+\left|\delta_{2}\right|\left(\frac{w_{0}\left(g_{1}(t) t\right)+w_{0}(t)}{1-w_{0}(t)}\right)^{2}\right) \frac{\int_{0}^{1} v\left(g_{2}(t) t\right) d \theta}{1-w_{0}(t)} g_{2}(t)
\end{aligned}
$$

and $h_{i}(t)=g_{i}(t)-1, i=1,2,3$, where $\delta_{1}, \delta_{2} \in \mathbb{R}$. Suppose that

$$
v(0)<3
$$

We have that $h_{j}(0)=-1<0, j=2,3, h_{1}(0)=\frac{v(0)}{3}-1<0$ and $h_{i}(t) \longrightarrow+\infty$ as $t \longrightarrow r_{0}^{-}$. It follows by the intermediate value theorem that functions $h_{i}$ have zeros in the interval $\left(0, r_{0}\right)$. Denote by $r_{i}$ the smallest zeros of functions $h_{i}$, respectively. Define the radius of convergence $r$ by

$$
r=\min \left\{r_{i}\right\}, \quad i=1,2,3
$$

Then, we have that for each $t \in[0, r) 0 \leq g_{i}(t)<1$.

Let $U(y, \rho), \bar{U}(y, \rho)$ denote respectively the open and closed balls in $X$ with center $y \in X$ and of radius $\rho>0$. Next, we present the local convergence analysis of method (1.7) using the preceding notation.

Theorem 1. Let $F: \Omega \subseteq X \longrightarrow Y$ be a continuously Fréchet differentiable operator and $\delta_{1}, \delta_{2}$ be two real parameters. Suppose: there exist $x^{*} \in \Omega$ and $w_{0}:[0,+\infty) \longrightarrow[0,+\infty)$ nondecreasing with $w_{0}(0)=0$ such that for each $x \in D$

$$
\begin{aligned}
& F\left(x^{*}\right)=0, \quad F^{\prime}\left(x^{*}\right)^{-1} \in L(Y, X), \\
& \left\|F^{\prime}\left(x^{*}\right)^{-1}\left(F^{\prime}(x)-F^{\prime}\left(x^{*}\right)\right)\right\| \leq w_{0}\left(\left\|x-x^{*}\right\|\right) ;
\end{aligned}
$$


there exist functions $w:[0,+\infty) \longrightarrow[0,+\infty), v:[0,+\infty) \longrightarrow(0,+\infty)$ with $w(0)=0$ such that for each $x, y \in \Omega_{0}:=\Omega \cap U\left(x^{*}, r_{0}\right)$

$$
\begin{aligned}
& \left\|F^{\prime}\left(x^{*}\right)^{-1}\left(F^{\prime}(x)-F^{\prime}(y)\right)\right\| \leq w(\|x-y\|), \\
& \left\|F^{\prime}\left(x^{*}\right)^{-1} F^{\prime}(x)\right\| \leq v\left(\left\|x-x^{*}\right\|\right)
\end{aligned}
$$

and (2.2) holds, $\bar{U}\left(x^{*}, r\right) \subseteq \Omega$, where $r_{0}, r$ are defined by (2.1) and (2.3), respectively. Then, the sequence $\left\{x_{n}\right\}$ generated by method (1.7) for $x_{0} \in$ $U\left(x^{*}, r\right)-\left\{x^{*}\right\}$ is well defined, remains in $U\left(x^{*}, r\right)$ for each $n=0,1,2, \ldots$ and converges to $x^{*}$. Moreover, the following estimates hold

$$
\begin{aligned}
& \left\|u_{n}-x^{*}\right\| \leq g_{1}\left(\left\|x_{n}-x^{*}\right\|\right)\left\|x_{n}-x^{*}\right\| \leq\left\|x_{n}-x^{*}\right\|<r, \\
& \left\|z_{n}-x^{*}\right\| \leq g_{2}\left(\left\|x_{n}-x^{*}\right\|\right)\left\|x_{n}-x^{*}\right\| \leq\left\|x_{n}-x^{*}\right\| \\
& \left\|x_{n+1}-x^{*}\right\| \leq g_{3}\left(\left\|x_{n}-x^{*}\right\|\right)\left\|x_{n}-x^{*}\right\| \leq\left\|x_{n}-x^{*}\right\| .
\end{aligned}
$$

Furthermore, the point $x^{*}$ is the only solution of equation $F(x)=0$ in $\Omega_{1}=$ $\Omega \cap U\left(x^{*}, r\right)$.

Proof. We shall show using mathematical induction that the sequence $\left\{x_{n}\right\}$ is well defined in $U\left(x^{*}, r\right)$ and converges to $x^{*}$ so that estimates (2.8)-(2.10) are satisfied. By hypothesis $x_{0} \in U\left(x^{*}, r\right)-\left\{x^{*}\right\},(2.1),(2.4)$ and (2.5) we get that

$$
\left\|F^{\prime}\left(x^{*}\right)^{-1}\left(F^{\prime}\left(x_{0}\right)-F^{\prime}\left(x^{*}\right)\right)\right\| \leq w_{0}\left(\left\|x_{0}-x^{*}\right\|\right) \leq w_{0}(r)<1 .
$$

It follows from (2.11) and the Banach perturbation lemma $[1,3,8]$ that $F^{\prime}\left(x_{0}\right)^{-1} \in L(Y, X)$,

$$
\left\|F^{\prime}\left(x_{0}\right)^{-1} F^{\prime}\left(x^{*}\right)\right\| \leq \frac{1}{1-w_{0}\left(\left\|x_{0}-x^{*}\right\|\right)}
$$

and $u_{0}$ is well defined by the first substep of method (1.7) for $n=0$. In view of (2.4) and (2.7), we get that

$$
F\left(x_{0}\right)=F\left(x_{0}\right)-F\left(x^{*}\right)=\int_{0}^{1} F^{\prime}\left(x^{*}+\theta\left(x_{0}-x^{*}\right)\right)\left(x_{0}-x^{*}\right) d \theta
$$

so,

$$
\begin{aligned}
\left\|F^{\prime}\left(x^{*}\right)^{-1} F\left(x_{0}\right)\right\| & =\left\|\int_{0}^{1} F^{\prime}\left(x^{*}\right)^{-1} F^{\prime}\left(x^{*}+\theta\left(x_{0}-x^{*}\right)\right)\left(x_{0}-x^{*}\right) d \theta\right\| \\
& \leq \int_{0}^{1} v\left(\theta\left\|x_{0}-x^{*}\right\|\right)\left\|x_{0}-x^{*}\right\| d \theta
\end{aligned}
$$

where we also used that $x^{*}+\theta\left(x_{0}-x^{*}\right) \in U\left(x^{*}, r\right)$, since

$$
\left\|x^{*}+\theta\left(x_{0}-x^{*}\right)-x^{*}\right\|=\theta\left\|x_{0}-x^{*}\right\|<r .
$$

Using the first substep of method (1.7) for $n=0$ and (2.4), we can write

$$
u_{0}-x^{*}=x_{0}-x^{*}-F^{\prime}\left(x_{0}\right)^{-1} F\left(x_{0}\right)+\frac{1}{3} F^{\prime}\left(x_{0}\right)^{-1} F\left(x_{0}\right) .
$$


Then, by (2.3), (2.4), (2.6), (2.12), (2.13) and (2.14) we obtain in turn that

$$
\begin{aligned}
\left\|u_{0}-x^{*}\right\| & \leq\left\|F^{\prime}\left(x_{0}\right)^{-1} F^{\prime}\left(x^{*}\right)\right\| \| F^{\prime}\left(x^{*}\right)^{-1} \int_{0}^{1}\left[F^{\prime}\left(x^{*}+\theta\left(x_{0}-x^{*}\right)\right)\right. \\
& \left.-F^{\prime}\left(x_{0}\right)\right]\left(x_{0}-x^{*}\right) d \theta\left\|+\frac{1}{3}\right\| F^{\prime}\left(x_{0}\right)^{-1} F^{\prime}\left(x^{*}\right)\|\| F^{\prime}\left(x^{*}\right)^{-1} F\left(x_{0}\right) \| \\
\leq & \frac{1}{1-w_{0}\left(\left\|x_{0}-x^{*}\right\|\right)} \int_{0}^{1} w\left((1-\theta)\left\|x_{0}-x^{*}\right\|\right) d \theta\left\|x_{0}-x^{*}\right\| \\
& \quad+\frac{\int_{0}^{1} v\left(\theta\left\|x_{0}-x^{*}\right\|\right) d \theta\left\|x_{0}-x^{*}\right\|}{3\left(1-w_{0}\left(\left\|x_{0}-x^{*}\right\|\right)\right)} \\
= & g_{1}\left(\left\|x_{0}-x^{*}\right\|\right)\left\|x_{0}-x^{*}\right\|<\left\|x_{0}-x^{*}\right\|<r,
\end{aligned}
$$

which shows (2.8) for $n=0$, and $u_{0} \in U\left(x^{*}, r\right)$. By the second substep of method (1.7) we can write that

$$
\begin{aligned}
z_{0}- & x^{*}=x_{0}-x^{*}-F^{\prime}\left(x_{0}\right)^{-1} F\left(x_{0}\right) \\
+ & \frac{3}{4}\left(I-\frac{3}{2} A\left(x_{0}\right)+\delta_{1} A\left(x_{0}\right)^{2}\right) A\left(x_{0}\right) F^{\prime}\left(x_{0}\right)^{-1} F\left(x_{0}\right) .
\end{aligned}
$$

We get that

$$
\begin{aligned}
\left\|A\left(x_{0}\right)\right\|= & \left\|F^{\prime}\left(x_{0}\right)^{-1}\left(F^{\prime}\left(u_{0}\right)-F^{\prime}\left(x_{0}\right)\right)\right\| \\
\leq & \left\|F^{\prime}\left(x_{0}\right)^{-1} F^{\prime}\left(x^{*}\right)\right\|\left[\left\|F^{\prime}\left(x^{*}\right)^{-1}\left(F^{\prime}\left(u_{0}\right)-F^{\prime}\left(x^{*}\right)\right)\right\|\right. \\
& \left.+\left\|F^{\prime}\left(x^{*}\right)^{-1}\left(F^{\prime}\left(x_{0}\right)-F^{\prime}\left(x^{*}\right)\right)\right\|\right] \\
\leq & \frac{w_{0}\left(\left\|u_{0}-x^{*}\right\|\right)+w_{0}\left(\left\|x_{0}-x^{*}\right\|\right)}{1-w_{0}\left(\left\|x_{0}-x^{*}\right\|\right)} \\
\leq & \frac{w_{0}\left(g_{1}\left(\left\|x_{0}-x^{*}\right\|\right)\left\|x_{0}-x^{*}\right\|\right)+w_{0}\left(\left\|x_{0}-x^{*}\right\|\right)}{1-w_{0}\left(\left\|x_{0}-x^{*}\right\|\right)} .
\end{aligned}
$$

Then, by (2.3), (2.4), (2.15)-(2.17) we have that

$$
\begin{aligned}
\| z_{0}- & x^{*}\|\leq\| x_{0}-x^{*}-F^{\prime}\left(x_{0}\right)^{-1} F\left(x_{0}\right) \| \\
+ & \frac{3}{4}\left(\|I\|+\frac{3}{2}\left\|A\left(x_{0}\right)\right\|+\left|\delta_{1}\right|\left\|A\left(x_{0}\right)\right\|^{2}\right)\left\|A\left(x_{0}\right)\right\|\left\|F^{\prime}\left(x_{0}\right)^{-1} F\left(x_{0}\right)\right\| \\
\leq & \frac{1}{1-w_{0}\left(\left\|x_{0}-x^{*}\right\|\right)} \int_{0}^{1} w\left((1-\theta)\left\|x_{0}-x^{*}\right\|\right) d \theta\left\|x_{0}-x^{*}\right\| \\
+ & \frac{3}{4}\left(1+\frac{3}{2} \frac{w_{0}\left(g_{1}\left(\left\|x_{0}-x^{*}\right\|\right)\left\|x_{0}-x^{*}\right\|\right)+w_{0}\left(\left\|x_{0}-x^{*}\right\|\right)}{1-w_{0}\left(\left\|x_{0}-x^{*}\right\|\right)}\right. \\
& \left|\delta_{1}\right|\left(\frac{w_{0}\left(g_{1}\left(\left\|x_{0}-x^{*}\right\|\right)\left\|x_{0}-x^{*}\right\|\right)+w_{0}\left(\left\|x_{0}-x^{*}\right\|\right)}{1-w_{0}\left(\left\|x_{0}-x^{*}\right\|\right)}\right) \\
& \times \frac{w_{0}\left(g_{1}\left(\left\|x_{0}-x^{*}\right\|\right)\left\|x_{0}-x^{*}\right\|\right)+w_{0}\left(\left\|x_{0}-x^{*}\right\|\right)}{1-w_{0}\left(\left\|x_{0}-x^{*}\right\|\right)} \\
& \times \frac{\int_{0}^{1} v\left(\theta\left\|x_{0}-x^{*}\right\|\right)\left\|x_{0}-x^{*}\right\| d \theta}{1-w_{0}\left(\left\|x_{0}-x^{*}\right\|\right)} \\
= & g_{2}\left(\left\|x_{0}-x^{*}\right\|\right)\left\|x_{0}-x^{*}\right\|<\left\|x_{0}-x^{*}\right\|<r,
\end{aligned}
$$


which shows $(2.9)$ for $n=0$ and $z_{0} \in U\left(x^{*}, r\right)$. Similarly, by (2.4) and the last substep of method (1.7) for $n=0$, we get that

$$
\begin{aligned}
\left\|x_{1}-x^{*}\right\| \leq & \left\|z_{0}-x^{*}\right\| \\
& +\left(1+\frac{3}{2}\left\|A\left(x_{0}\right)\right\|+\left|\delta_{2}\right|\left\|A\left(x_{0}\right)\right\|^{2}\right)\left\|F^{\prime}\left(x_{0}\right)^{-1} F\left(z_{0}\right)\right\| \\
\leq & g_{2}\left(\left\|x_{0}-x^{*}\right\|\right)\left\|x_{0}-x^{*}\right\| \\
& +\left(1+\frac{3}{2} \frac{w_{0}\left(g_{1}\left(\left\|x_{0}-x^{*}\right\|\right)\left\|x_{0}-x^{*}\right\|\right)+w_{0}\left(\left\|x_{0}-x^{*}\right\|\right)}{1-w_{0}\left(\left\|x_{0}-x^{*}\right\|\right)}\right. \\
& \left.+\left|\delta_{2}\right|\left(\frac{w_{0}\left(g_{1}\left(\left\|x_{0}-x^{*}\right\|\right)\left\|x_{0}-x^{*}\right\|\right)+w_{0}\left(\left\|x_{0}-x^{*}\right\|\right)}{1-w_{0}\left(\left\|x_{0}-x^{*}\right\|\right)}\right)^{2}\right) \\
& \times \frac{\int_{0}^{1} v\left(\theta g_{2}\left(\left\|x_{0}-x^{*}\right\|\right)\left\|x_{0}-x^{*}\right\|\right) g_{2}\left(\left\|x_{0}-x^{*}\right\|\right) d \theta}{1-w_{0}\left(\left\|x_{0}-x^{*}\right\|\right)}\left\|x_{0}-x^{*}\right\| \\
= & g_{3}\left(\left\|x_{0}-x^{*}\right\|\right)\left\|x_{0}-x^{*}\right\|<\left\|x_{0}-x^{*}\right\|<r,
\end{aligned}
$$

which shows (2.10) for $n=0$ and $x_{1} \in U\left(x^{*}, r\right)$, where we also used (2.13) for $z_{0}=x_{0}$. By simply replacing $u_{0}, u_{0}, z_{0}, x_{1}$ by $x_{k}, u_{k}, z_{k}, x_{k+1}$ in the preceding estimates, we arrive at (2.8)-(2.10). Then, from the estimate

$$
\left\|x_{k+1}-x^{*}\right\| \leq c\left\|x_{k}-x^{*}\right\|<r, c=g_{3}\left(\left\|x_{0}-x^{*}\right\|\right) \in[0,1),
$$

we deduce that $\lim x_{k}=x^{*}$ and $x_{k+1} \in U\left(x^{*}, r\right)$. Finally, to show the uniqueness part, let $T=\int_{0}^{1} F^{\prime}\left(x^{*}+\theta\left(y^{*}-x^{*}\right)\right) d \theta$, where $F\left(y^{*}\right)=0$ and $y^{*} \in \Omega_{1}$. Using (2.5), we get that

$$
\left\|F^{\prime}\left(x^{*}\right)^{-1}\left(T-F^{\prime}\left(x^{*}\right)\right)\right\| \leq \int_{0}^{1} v\left(\theta\left\|x^{*}-y^{*}\right\|\right) d \theta<1 .
$$

That is $T^{-1} \in L(Y, X)$. Then from the identity

$$
0=F\left(y^{*}\right)-F\left(x^{*}\right)=T\left(y^{*}-x^{*}\right)
$$

we conclude that $x^{*}=y^{*}$.

Remark 1.

1. In view of $(2.5)$ and the estimate

$$
\begin{aligned}
\left\|F^{\prime}\left(x^{*}\right)^{-1} F^{\prime}(x)\right\| & =\left\|F^{\prime}\left(x^{*}\right)^{-1}\left(F^{\prime}(x)-F^{\prime}\left(x^{*}\right)\right)+I\right\| \\
& \leq 1+\left\|F^{\prime}\left(x^{*}\right)^{-1}\left(F^{\prime}(x)-F^{\prime}\left(x^{*}\right)\right)\right\| \leq 1+w_{0}\left(\left\|x-x^{*}\right\|\right)
\end{aligned}
$$

condition (2.7) can be dropped and $v$ can be replaced by $v(t)=1+w_{0}(t)$.

2. Let $w_{0}(t)=L_{0} t, w(t)=L t, v(t)=M$ for some $L_{0}>0, L>0$ and $M \geq 1$. In this special case, the results obtained here can be used for operators $F$ satisfying autonomous differential equations [6] of the form

$$
F^{\prime}(x)=P(F(x)),
$$

where $P: Y \longrightarrow Y$ is a continuous operator. Then, since $F^{\prime}\left(x^{*}\right)=$ $P\left(F\left(x^{*}\right)\right)=P(0)$, we can apply the results without actually knowing $x^{*}$. For example, let $F(x)=e^{x}-1$. Then, we can choose: $P(x)=x+1$. 
3. The radius $r^{*}=2 /\left(2 L_{0}+L\right)$ was shown by us to be the convergence radius of Newton's method $[4,5]$

$$
x_{n+1}=x_{n}-F^{\prime}\left(x_{n}\right)^{-1} F\left(x_{n}\right) \text { for each } n=0,1,2, \ldots
$$

under the conditions (2.4)-(2.7). It follows from the definition of $r$ that the convergence radius $r$ of the method (1.7) cannot be larger than the convergence radius $r^{*}$ of the second order Newton's method (2.18).

As already noted in $[4,5] r^{*}$ is at least as large as the convergence ball given by Rheinboldt [8] $r_{R}=\frac{2}{3 L}$. In particular, for $L_{0}<L$ we have that $r_{R}<r^{*}$ and

$$
\frac{r_{R}}{r^{*}} \rightarrow \frac{1}{3} \quad \text { as } \quad \frac{L_{0}}{L} \rightarrow 0 .
$$

That is our convergence ball $r^{*}$ is at most three times larger than Rheinboldt's. The same value for $r_{R}$ was given by Traub [9].

4. It is worth noticing that method (1.7) is not changing when we use the conditions of Theorem 1 instead of the stronger conditions used in $[2,7,10$, 11]. Moreover, we can compute the computational order of convergence (COC) defined by

$$
\xi=\ln \left(\frac{\left\|x_{n+1}-x^{*}\right\|}{\left\|x_{n}-x^{*}\right\|}\right) / \ln \left(\frac{\left\|x_{n}-x^{*}\right\|}{\left\|x_{n-1}-x^{*}\right\|}\right)
$$

or the approximate computational order of convergence

$$
\xi_{1}=\ln \left(\frac{\left\|x_{n+1}-x_{n}\right\|}{\left\|x_{n}-x_{n-1}\right\|}\right) / \ln \left(\frac{\left\|x_{n}-x_{n-1}\right\|}{\left\|x_{n-1}-x_{n-2}\right\|}\right) .
$$

This way we obtain in practice the order of convergence in a way that avoids the bounds involving estimates using estimates higher than the first Fréchet derivative of operator $F$.

\section{$3 \quad$ Numerical Examples}

The numerical examples are presented in this section.

Example 1. Let $X=Y=\mathbb{R}^{3}, D=\bar{U}(0,1), x^{*}=(0,0,0)^{T}$. Define function $F$ on $D$ for $w=(x, y, z)^{T}$ by

$$
F(w)=\left(e^{x}-1, \frac{e-1}{2} y^{2}+y, z\right)^{T} .
$$

Then, the Fréchet-derivative is given by

$$
F^{\prime}(v)=\left[\begin{array}{ccc}
e^{x} & 0 & 0 \\
0 & (e-1) y+1 & 0 \\
0 & 0 & 1
\end{array}\right]
$$

Notice that using the (2.5)-(2.7) conditions, we get $L_{0}=e-1, L=e^{\frac{1}{L_{0}}}=M$, so $w_{0}(t)=L_{0} t=(e-1) t, w(t)=L t=e^{\frac{1}{L_{0}}} t$ and $v(t)=M=e^{\frac{1}{L_{0}}}$. Then for $\delta_{1}=\delta_{2}=0.5$, the parameters are

$$
r_{1}=0.1544, r_{2}=0.1422=r, r_{3}=0.1592 .
$$


Example 2. Let $X=Y=C[0,1]$, the space of continuous functions defined on $[0,1]$ equipped with the max norm. Let $D=\bar{U}(0,1)$. Define function $F$ on $D$ by

$$
F(\varphi)(x)=\varphi(x)-5 \int_{0}^{1} x \theta \varphi(\theta)^{3} d \theta
$$

We have that

$$
F^{\prime}(\varphi(\xi))(x)=\xi(x)-15 \int_{0}^{1} x \theta \varphi(\theta)^{2} \xi(\theta) d \theta, \text { for each } \xi \in D .
$$

Then, we get that $x^{*}=0, L_{0}=7.5, L=15, M=2$ so $w_{0}(t)=7.5 t, w(t)=15 t$ and $v(t)=2$. Then for $\delta_{1}=\delta_{2}=0.5$, the parameters are

$$
r_{1}=0.0219, r=r_{2}=r_{3}=0.0002 \text {. }
$$

Example 3. Returning back to the motivational example at the introduction of this study, we have $w_{0}(t)=w(t)=\frac{1}{8}\left(\frac{3}{2} \sqrt{t}+t\right)$ and $v(t)=1+w_{0}(t)$. Then for $\delta_{1}=\delta_{2}=0.5$, the parameters are

$$
r_{1}=2.1863, r=r_{2}=r_{3}=0.1297 \text {. }
$$

\section{References}

[1] I.K. Argyros. Computational theory of iterative methods, volume 15 of Studies in Computational Mathematics. Elsevier Science, San Diego, USA, 2007.

[2] I.K. Argyros, D. Chen and Q. Qian. The Jarratt method in Banach space setting. Journal of Computational and Applied Mathematics, 51(1):103-106, 1994. https://doi.org/10.1016/0377-0427(94)90093-0.

[3] I.K. Argyros and S. Hilout. Computational methods in nonlinear analysis. Efficient algorithms, fixed point theory and applications. World Scientific, 2013.

[4] V. Candela and A. Marquina. Recurrence relations for rational cubic methods I: The Halley method. Computing, 44(2):169-184, 1990. https://doi.org/10.1007/BF02241866.

[5] V. Candela and A. Marquina. Recurrence relations for rational cubic methods II: The Chebyshev method. Computing, 45(4):355-367, 1990. https://doi.org/10.1007/BF02238803.

[6] M.A. Hernández and M.A. Salanova. Sufficient conditions for semilocal convergence of a fourth order multipoint iterative method for solving equations in Banach spaces. Southwest Journal of Pure and Applied Mathematics [electronic only], 1:29-40, 1999.

[7] P. Jarratt. Some fourth order multipoint iterative methods for solving equations. Mathematics of Computation, 20(95):434-437, 1996. https://doi.org/10.2307/2003602.

[8] W. Rheinboldt. An adaptive continuation process for solving systems of nonlinear equations, volume 3. Banach Center Publications, 1978.

[9] J.F. Traub. Iterative methods for the solution of equations. AMS Chelsea Publishing. American Mathematical Society, 1982. 
[10] X. Wang and J. Kou. $R$ - order of convergence for modified Jarratt method with less computation of inversion. Appl. Math. Comput., (To appear), 2017.

[11] X. Wang, J. Kou and C. Gu. Semilocal convergence of a sixth-order Jarratt method in Banach spaces,. Numerical Algorithms, 57(4):441-456, 2011. https://doi.org/10.1007/s11075-010-9438-1. 